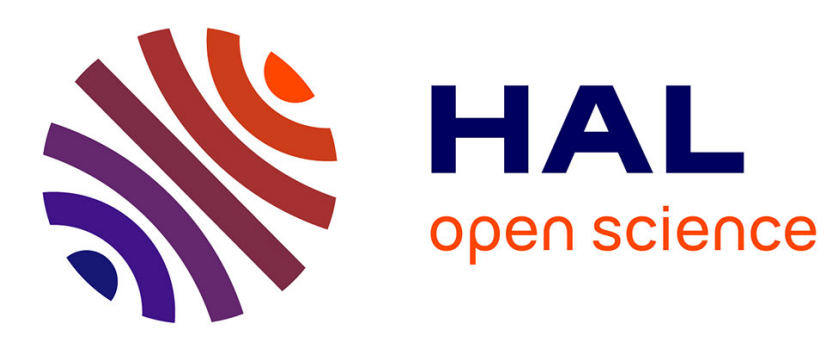

\title{
Les formes de travail scientifique en STAPS : entre disciplinarité et interdisciplinarité
}

\author{
Cécile Collinet, Philippe Terral, Patrick Trabal
}

\section{To cite this version:}

Cécile Collinet, Philippe Terral, Patrick Trabal. Les formes de travail scientifique en STAPS : entre disciplinarité et interdisciplinarité. Movement \& Sport Sciences - Science \& Motricité, 2011, 94, pp. 41-53. 10.1051/sm/2015031 . hal-01471598

\section{HAL Id: hal-01471598 \\ https://hal.parisnanterre.fr/hal-01471598}

Submitted on 18 Dec 2020

HAL is a multi-disciplinary open access archive for the deposit and dissemination of scientific research documents, whether they are published or not. The documents may come from teaching and research institutions in France or abroad, or from public or private research centers.
L'archive ouverte pluridisciplinaire HAL, est destinée au dépôt et à la diffusion de documents scientifiques de niveau recherche, publiés ou non, émanant des établissements d'enseignement et de recherche français ou étrangers, des laboratoires publics ou privés. 


\title{
Les formes de travail scientifique en STAPS : entre disciplinarité et interdisciplinarité
}

\author{
Cécile Collinet ${ }^{1}$, Philippe Terral ${ }^{2}$ et Patrick Trabal ${ }^{3}$ \\ 1 Université Paris-Est Marne-La-Vallée, ACP EA 3350, 5 bd Descartes, 77454 Champs-sur-Marne, France \\ 2 Université Paul Sabatier, laboratoire PRISSMH, 118 route de Narbonne, 31062 Toulouse cedex 9, France \\ 3 Université Paris-Ouest Nanterre La Défense, laboratoire CERSM, 200 avenue de la République, 92000 Nanterre, France
}

Reçu le 22 avril 2015 - Accepté le 7 octobre 2015

\begin{abstract}
Résumé. Les STAPS s'inscrivent dans un vaste mouvement à la fois scientifique et politique encourageant l'interdisciplinarité. Considérée comme productrice de connaissances originales ou davantage heuristiques pour traiter les questions sociales complexes, sa mise en place n'est pas sans poser de problèmes dans le travail scientifique ordinaire. Cette discipline, originellement pluridisciplinaire, est imprégnée par la question interdisciplinaire. À partir d'un corpus de textes variés et d'entretiens avec des enseignants-chercheurs, notre enquête vise à mieux comprendre les modalités de l'activité interdisciplinaire. Nous soulignons la tension entre deux injonctions propres au travail interdisciplinaire et parfois contradictoires : l'interdisciplinarité et l'exigence académique disciplinaire. Nous montrons que c'est au travers de six formes de travail que nous présenterons comme des chemins frayés, entendus comme des régimes d'action stabilisés, que se dessinent les modes de travail interdisciplinaire repérés dans le domaine des STAPS.
\end{abstract}

Mots clés : STAPS, interdisciplinarité, exigences disciplinaires, chemins frayés

\begin{abstract}
The forms of scientific work in STAPS: between disciplarity and interdisciplinarity.

The STAPS follow a vast movement which is both political and scientific, and which encourages interdisciplinarity. Considered as able to produce specific but also heuristic knowledge in order to answer complex social questions, its implementation can be problematic for the usual scientific work. This discipline, originally pluridisciplinary, is fully related to interdisciplinarity. Based on a group of various texts and interviews with teachers-researchers, our study aims to a better understanding of the conditions of interdisciplinary activity. We underline tension, and sometimes contradictions between two injunctions related to interdisciplinary work: interdisciplinarity and academic disciplinary needs. We show that it is through six different types of work which we introduce as paths, understood as stabilized action regimes, that ways of interdisciplinary work in the field of STAPS can be observed.
\end{abstract}

Key words: STAPS, interdisciplinarity, academic disciplinary needs, paths

\section{Introduction}

L'interdisciplinarité est un sujet classique qui mobilise la communauté scientifique de manière récurrente. On peut situer un renforcement du questionnement à son sujet dans la deuxième moitié du $20^{\mathrm{e}}$ siècle. C'est à la faveur des réformes de l'enseignement universitaire, au cours des années 1960 et 1970, qu'elle s'est imposée comme thème de réflexion quasi incontournable du renouveau de l'université. L'avènement de la notion d'interdisciplinarité est concomitant du mouvement de contestation sociale qui portait en creux une critique de l'hyperspécialisation, et de l'approche mono-disciplinaire, de la division du savoir en disciplines étanches et de leur hiérarchie qui structuraient l'organisation de la formation universitaire. Dans les années 1970, des organismes internationaux comme l'UNESCO ou l'OCDE (1972) entretiennent le débat. Régulièrement depuis, les programmes et politiques concernant la recherche la mettent en avant.

Le domaine des STAPS est particulièrement concerné par la question de l'interdisciplinarité depuis son origine dans les années 1980. Fondée sur une pluralité de 
disciplines tant scientifiques que technologiques ou pratiques, elle s'est très tôt posée la question des relations entre ces divers secteurs. Dès 1981, à la toute naissance des STAPS, Jacques Thibault, un des premiers enseignants-chercheurs de la filière écrivait :

\begin{abstract}
«Les APS constituent par leur diversité un carrefour pluridisciplinaire ou dans le meilleur des cas, interdisciplinaire, c'est-à-dire tenant compte des influences réciproques des diverses disciplines. Enseigner les STAPS, quelle que soit son origine, c'est donc obligatoirement avoir la préoccupation de ne pas réduire la relation éducative ou le fait éducatif pris dans un sens très large, à une seule composante : la sienne »(Thibault, 1981).
\end{abstract}

S'interroger sur l'interdisciplinarité c'est donc s'engager dans un débat qui a largement occupé la scène dans le domaine des STAPS et qui, aujourd'hui encore, interroge (Collinet, Terral \& Trabal, 2012, Escriva, 2008, Vanpoulle, 2011).

Une première voie pour avancer sur cette question serait de tenter de définir ce que pourrait désigner ce terme et d'en cerner les contours. Les définitions ne manquent pas. Dès les années 1970, le travail du séminaire de Nice organisé par l'OCDE (OECD, 1972) a mis au jour une pluralité de définitions possibles en jouant sur des terminologies variées et des qualifications sophistiquées : interdisciplinarité hétérogène, pseudointerdisciplinarité, interdisciplinarité auxiliaire, composite, complémentaire, unificatrice (Heckhausen, 1972), linéaire, structurale, restrictive (Boisot, 1972) . . La modélisation proposée par Piaget (1972) a retenu l'attention et a souvent été mobilisée dans la littérature. L'auteur établit une distinction hiérarchique, de trois niveaux, basée sur le degré d'intégration des interactions entre les disciplines : le niveau multidisciplinaire, le niveau interdisciplinaire et enfin le niveau transdisciplinaire. Plus récemment, d'autres auteurs ont tenté de circonscrire la notion (Sinaceur, 1992; Sperber, 2010). L'enjeu est alors souvent de construire des catégories permettant de ranger les modes de travail scientifique dans des formes diversifiées d'interdisciplinarité.

Notre projet est ici différent et s'éloigne d'une épistémologie normative qui se contenterait de définir les règles $\mathrm{du}$ travail interdisciplinaire et se rapproche de travaux plus empiriques comme ceux de Vinck (2000) ou de Prud'homme, Gingras, Couillard et Terrasson (2012), en s'intéressant à la manière dont les acteurs la définissent, la discutent et le mettent en œuvre dans leurs productions et leur travail scientifiques. Il ne s'agit pas de proposer une modélisation épistémologique théorique, certes intellectuellement satisfaisante, mais souvent décontextualisée des pratiques mais plutôt de repérer comment l'invitation voire l'injonction à l'interdisciplinarité prend forme dans les activités scientifiques.

Notre projet est de mettre au jour les tensions qu'elle suscite et les formes qu'elle peut prendre dans le travail scientifique en STAPS dans son écriture, la constitution de ses collectifs de recherche ou ses évaluations.

Pour mener à bien ce projet, nous fondons notre travail sur des corpus de textes de nature différente. Nous analysons les productions des chercheurs au travers de leurs écrits scientifiques dans des revues propres à la $74^{\mathrm{e}}$ section en considérant les articles de la revue STAPS $(n=602)$ parus de 1980 à 2010, de la revue Sciences et motricité entre 2002 et $2009^{1}(n=143)$ ainsi que des actes de congrès de l'Association des Chercheurs en Activités Physiques et Sportives (ACAPS) $(n=1828)$, structure qui porte la précédente revue et qui se réunit en colloques tous les deux ans depuis 1995. Nous avons complété ce corpus de 29 rapports émis par l'AERES ${ }^{2}$ portant sur les laboratoires en STAPS sur la période de 2008-20093 . Enfin, 20 entretiens ont aussi été réalisés auprès d'enseignants-chercheurs en STAPS ${ }^{4}$. Nous pensons que la complémentarité de ce matériau permet de saisir à la fois l'expression des difficultés du travail interdisciplinaire, les façons dont celui se met en scène dans les articles et les évaluations qui en découlent.

D'un point de vue méthodologique, nos corpus ont été analysés avec le logiciel Prospéro. Développé à l'origine dans le cadre d'une sociologie des controverses (Chateauraynaud, 2003), il résulte d'une confrontation des apports de la statistique textuelle (calculs des fréquences et de cooccurrences multiples), de la linguistique (analyse des énoncés et des enchaînements discursifs), de l'intelligence artificielle (système de raisonnement et d'inférence), des analyses de réseaux (calculs d'associations et de graphes) et de la sociologie cognitive (étude des procédés de codage et d'interprétation). Il est notamment capable de mettre en perspective la dimension historique du dossier à laquelle les acteurs sociaux font référence (par exemple lorsqu'ils mentionnent des positions frayées) et de confronter les catégories d'analyse de l'utilisateur non seulement à des propriétés émergentes mais aussi à des

\footnotetext{
1 Cette période a été guidée par la disponibilité des articles en ligne.

2 Agence d'Évaluation de la Recherche et de l'Enseignement remplacée aujourd'hui par le Haut Conseil de l'Évaluation de la Recherche et de l'Enseignement Supérieur.

3 Nous avons fait le choix de nous focaliser sur une campagne d'évaluation pour plus de facilité compte tenu de la densité totale du corpus.

${ }^{4}$ Les entretiens concernent des maîtres de conférences et des professeurs dont certains directeurs de laboratoire. L'échantillon couvre toutes les disciplines scientifiques représentées en STAPS (les disciplines nous ont été données par les interviewés eux-mêmes) : 3 enseignants-chercheurs en psychologie, 3 en science du mouvement humain, 2 en sociologie, 2 en didactique 2 en praxéologie, 3 en physiologie, 1 en neurosciences, 1 en biomécanique, 3 en histoire; $5 \mathrm{MCF}$ et 15 professeurs. Ces entretiens portent sur la définition de leur discipline, sur leurs modes de travail scientifique et leur rapport à l'interdisciplinarité. Nous avons essayé d'avoir une variété de disciplines, la méthode de proche en proche ne nous a pas permis d'avoir un échantillon représentatif.
} 
propriétés singulières à partir desquelles s'opèrent des démarcations, des différenciations, voire des ruptures décisives. Il apparaît du coup comme particulièrement adapté pour identifier les tensions et les arguments déployés dans les controverses d'anticipation, lorsqu'il s'agit d'envisager les conséquences d'une politique en matière de disciplinarité et d'interdisciplinarité.

Nous montrerons avant tout l'existence d'une tension fondamentale entre une exigence académique essentiellement disciplinaire et des tentatives (plus ou moins avancées) de mise en place d'une forme d'interdisciplinarité. Nous analyserons dans cet article combien cette double contrainte impacte les formes interdisciplinaires conduisant les chercheurs à se positionner sur un continuum marqué par des contraintes de disciplinarité et/ou d'interdisciplinarité plus ou moins fortes.

\section{Le travail scientifique : entre ancrages disciplinaires et velléités interdisciplinaires}

Si l'on en croit Weingart (2010), on peut repérer dès le début du $19^{\mathrm{e}}$ siècle une critique de la perte d'unité de la science. Pour lui, ce mouvement porteur d'appels à l'interdisciplinarité est initié par des philosophes des sciences et des biologistes dans les années 1930 et amplifié dans les années 1960-1970 notamment autour des réflexions menées par l'OCDE et du contexte de critique politique dont la science n'est pas exclue. Actuellement les politiques de recherche, positionnent le travail scientifique à l'interface d'une exigence disciplinaire d'excellence répondant notamment à l'organisation sociale de la science et d'une volonté d'ouverture interdisciplinaire présentée souvent comme une réponse mieux adaptée aux enjeux sociaux de la science ${ }^{5}$ et aux problèmes complexes posés par de nouveaux objets (le climat en étant un exemple typique). Cette double contrainte apparaît souvent dans les discours publics sur la science, y compris au plus haut niveau de l'État. L'exigence académique implique un repérage disciplinaire : "Parce qu'au cour du modèle universitaire, il y a l'exigence académique » (Valérie Pécresse, 13/05/20116). L'importance de l'interdisciplinarité est néanmoins souvent rappelée dans le cadre des modifications de la politique de la recherche

\footnotetext{
5 «Élisabeth de Turckheim considère qu'une "discipline seule ne peut pas répondre aux problèmes complexes posés par la société". En effet, l'adaptation au changement climatique, le vieillissement de la population et les pertes cognitives qui l'accompagnent ou encore l'utilisation ou non d'OGM sont autant de sujets très pointus mais complètement interdisciplinaires. Ils nécessitent la mobilisation de savoirs pointus dans des secteurs très différents comme la sociologie, l'écologie, la physique du globe, les sciences politiques ou encore l'économie !» Olivier Monot (2012) (http://www.letudiant.fr/educpros/actualite/ la-difficile-evaluation-de-la-recherche-interdisciplinaire.html).

${ }^{6}$ Précresse V., Discours de clôture du colloque CPU « une ambition, la Licence », 13/05/2011.
}

ou bien questionnée dans sa réelle faisabilité. Ainsi, par exemple, la mise en place des PRES était-elle parfois présentée comme une opportunité pour l'interdisciplinarité : «Ils [les organismes de recherche nationaux] assumeront alors des rôles qu'aucune d'entre elles [universités] ne peut jouer séparément : la programmation et le financement des grands projets interdisciplinaires de dimension nationale ou mondiale » (CPU, 03/07/2008 ${ }^{7}$ ). Le regroupement des établissements sous la forme de COMUE (Communauté d'Universités et d'Établissements, dont les statuts ont été créés en 2013) est souvent présenté aussi comme une opportunité pour structurer des projets interdisciplinaires comme les projets PEPS (Projet Exploratoire Premier Soutien de Site) par exemple ${ }^{8}$.

En revanche, la rationalisation évaluative orchestrée par l'AERES (dès 2006) est ressentie par certains comme un frein à l'interdisciplinarité en privilégiant des logiques disciplinaires d'excellence : «Comme conséquence des choix restrictifs imposés par l'AERES, on a noté, dans pas mal de cas, que des champs scientifiques entiers n'étaient pas couverts par les experts des comités, tout comme certaines dimensions pluridisciplinaires des activités de recherche »(C3N2, 04/03/2010). La politique de la recherche, pointant la volonté de reconnaissance internationale des travaux scientifiques ${ }^{9}$ d'une part, et l'intérêt pour des sujets de société complexes d'autre part, va interpeller l'interdisciplinarité. Cependant, les outils d'évaluation de la recherche entraînent pour leur part un repli sur les disciplines provoquant une tension qui est perceptible à de nombreux moments dans les discours (Trabal, Collinet, \& Terral, 2014).

\footnotetext{
7 www.cpu.fr/publication/position-de-la-cpu-sur-
} lorganisation-de-la-recherche-publique-en-Franceuniversites-et-organismes/.

8 « Au niveau territorial, où les politiques de site conduisent à des regroupements d'établissements, l'interdisciplinarité est souvent utilisée comme source et force de structuration, et parfois seule à jouer ce rôle. En tant que partenaire privilégié, le CNRS est moteur dans la mise en place d'actions visant à soutenir des projets interdisciplinaires exploratoires. Ces actions, nombreuses et variées, sont adaptées à chacun des sites en fonction des opportunités et des ressources. Elles se traduisent le plus souvent par le lancement d'appels à projets de type PEPS de site qui peuvent faire l'objet, l'année $N+1$, d'une évolution et/ou par de la mise en ouvre de projets ciblés. La procédure est très réactive pour faciliter un démarrage rapide des projets lauréats » (http://www.cnrs.fr/mi/spip.php? article8).

9 Notons que cette reconnaissance internationale peut passer par une disciplinarité stricte sur des objets très circonscrits ou par une recherche originale et interdisicplinaire : «L'interdisciplinarité ne doit pas être pour autant limitée à la recherche finalisée et appliquée. "C'est aussi à la croisée des disciplines que se situent les avancées", soutient Didier Houssin. Ces zones de rencontres entre les disciplines restent encore mystérieuses et attisent la curiosité des chercheurs » Olivier Monot (2012) http://www.letudiant.fr/educprosr/actualite/ la-difficile-evaluation-de-la-recherche-interdisciplinaire.html). 
L'interdisciplinarité suscite à la fois des revendications, des invitations voire des injonctions, mais fait également l'objet de difficultés et d'interrogations. Dans tous les cas, cette thématique jouxte celle de la disciplinarité. C'est d'ailleurs en grande partie ce qu'ont montré Stehr et Weingart (2000) lorsqu'ils avancent que l'interdisciplinarité est à la fois un processus externe et interne aux disciplines en liaison avec leur dynamique propre; dynamique qu'ils considèrent relativement constante depuis plus de deux siècles. D'un point de vue «externe », c'est tout d'abord, un appel persistant depuis le début du $19^{\mathrm{e}}$ siècle des acteurs critiquant la perte d'unité de la science face à la diversification et la multiplication des disciplines. Aujourd'hui, l'interdisciplinarité est promue par des agences visant à diriger les recherches vers des objectifs politiques désirés. On peut même parler de mots d'ordre voire d'injonctions. Au-delà de ces facteurs 《externes », l'interdisciplinarité renvoie également aux mouvements internes des disciplines qui révèlent des effets concomitants de structuration, de spécialisation et d'hybridation.

Lors de sa création, au début des années 1980, la toute jeune $74^{\mathrm{e}}$ section du CNU (STAPS), est fortement marquée par ces réflexions sur la politique des sciences. On peut même dire que la tension entre visions disciplinaires et interdisciplinaires est consubstantielle à la création des sciences du sport (Collinet, 2000; Terral, 2003). Le débat pionnier qui, dès les années 1970, a opposé Georges Vigarello à Pierre Parlebas, tous deux enseignants d'EPS ayant développé des recherches dans le domaine des sciences humaines et sociales, rend particulièrement compte de ce processus. La construction des STAPS est appréhendée entre une conception encourageant une forme de juxtaposition des disciplines qui tendent à se développer en lien étroit avec leur discipline-mère dans une sorte de séparatisme et une autre qui invite les disciplines à collaborer et à échanger tout en restant dans leur spécificité épistémologique (position qu'a défendue par exemple Vigarello; voir notamment son entretien dans l'ouvrage de Collinet, 2001), et enfin une dernière qui envisage une véritable transdisciplinarité entre des modèles qui, en se métissant, produisent une forme nouvelle de connaissance (comme la praxéologie motrice de Parlebas par exemple).

Comme pour l'ensemble de l'espace scientifique, l'interdisciplinarité en STAPS est donc source de revendications, d'invitations plus ou moins appuyées et suscite des interrogations voire des critiques. Celles-ci sont par exemple visibles dans les rapports évaluatifs des laboratoires de l'AERES. S'ils témoignent d'un intérêt certain pour l'interdisciplinarité ${ }^{10}$, nombreux sont ceux qui

\footnotetext{
10 L'interdisciplinarité est visible dans les comptes-rendus : «La pluridisciplinarité est assumée » (AERES, 01/04/2008), «L'équipe est résolument pluridisciplinaire » (AERES, 01/03/2009) ; «Cette interdisciplinarité irrigue la recherche menée par l'équipe»; «Les responsables de l'axe souhaitent de manière très ambitieuse relever un défi épistémologique capital pour les STAPS en valorisant une approche multidisciplinaire et transdisciplinaire $\gg(A E R E S, 1 / 01 / 2009)$. En outre, elle est
}

émettent un doute sur l'opérationnalité des approches proposées ou qui rappellent l'exigence disciplinaire :

«Si l'on peut trouver, sur certaines thématiques,
des équipes internationales plus pointues, on ne
trouvera cependant pas d'équivalent dans l'analyse
pluridisciplinaire d'un objet donné. C'est ainsi un
choix explicite de l'unité : favoriser l'interdiscipli-
narité et travailler sur les déterminants clefs plu-
tôt que de tracer un sillon profond sur un thème
donné qui ne sera peut être pas déterminant. Il fau-
dra cependant veiller à garder au cours du temps
un haut niveau de compétences disciplinaires, car
il y aurait risque de perdre toute pertinence dans
un travail pluiridisciplinaire » (Rapport, Janvier
$2011^{11}$ ).

En outre, nous avons pu observer dans nos entretiens que les chercheurs jouant le jeu de l'interdisciplinarité trouvent difficilement à se faire publier. Cette difficulté est vécue par les chercheurs dans leur positionnement de recherche, surtout par ceux qui se placent dans une posture interdisciplinaire revendiquée. Nos entretiens ont mis en évidence que les chercheurs s'attachent à publier leurs résultats dans des revues spécialisées en orientant clairement la présentation de leur travail dans un axe disciplinaire, même lorsque les travaux ont été réalisés dans un cadre interdisciplinaire. Lorsque les travaux se veulent originaux et interdisciplinaires, les chercheurs ont de nombreuses difficultés à surmonter comme cette chercheuse qui allie physiologie et psychologie dans le cadre de ses travaux :

«Il y a des contraintes déjà parce qu'on se fait critiquer des deux côtés. Quand je vais dans des congrès purement physiologiques, je me fais un peu critiquer parce que j'amène de la psycho, et pareil quand je vais dans des congrès de psycho je me fais critiquer parce que je ne suis pas estampillée psycho, donc si tu veux, c'est très dur parce qu'en France on a pas encore cette culture ... enfin moi j'ai commencé ça dans ma thèse en 199\%, et en STAPS en France c'était très rare. Et maintenant de plus en plus il y a des gens qui font ça, mais on n'est pas nombreux encore. [...] Mais même ici, enfin je veux dire quand je suis arrivée, je suis physiologiste, je suis à l'origine d'un diplôme de préparateur mental, je suis coach par ailleurs. Et donc les gens ne comprennent pas parce qu'ils n'arrivent pas à te mettre dans des cases »(Entretien 11).

souvent soulignée comme un élément positif : «Conclusions - Points forts : Il s'agit d'un projet scientifique de qualité, interdisciplinaire » (AERES, 01/03/2008), «La formation pluridisciplinaire est un plus pour ce laboratoire »(AERES, 01/03/2009).

11 La confidentialité des rapports a fait débat et nous préférons, ici, ne pas citer précisément les laboratoires concernés par les évaluations utilisées. 
Cette difficulté est clairement ressentie par de nombreux chercheurs :
«Je pense que oui, le contexte institutionnel quoique le discours peut être à double face parce qu'on a l'impression qu'il valorise plus les ap- proches interdisciplinaires mais en même temps, les lieux de publication ne sont pas interdiscipli- naires. C'est un problème » (Entretien 5).

Cette difficulté est rendue visible dans les rapports d'équipes :

\begin{abstract}
« Le groupe démontre une volonté appréciable et originale d'inscrire ses recherches en situation écologique et pluridisciplinaire. Néanmoins, la pluridisciplinarité affichée n'est pas toujours suivie d'effets dans les publications des chercheurs qui restent relativement disciplinaires et de ce fait émiettées. Il est vrai que les publications pluridisciplinaires ne sont pas toujours valorisées dans l'espace de publication offert aux chercheurs » (Rapport Novembre 2010).
\end{abstract}

Finalement, il semble que le travail interdisciplinaire et sa publication, si elle est privilégiée dans les discours politiques sur la science d'une part et par les grands projets liant science et société d'autre part, demeure un élément de risque pour le chercheur qui peut ne pas trouver dans ce type de recherche de possibilités de publication à la hauteur des critères scientifiques d'excellence. Les stratégies individuelles renvoient alors à la prudence d'une part et à l'assurance d'une réversibilité possible des choix interdisciplinaires vers une disciplinarité plus conventionnelle d'autre part :

«Globalement, les quelques publications qui sont sorties. Pour te donner un exemple, il y avait une manip et plusieurs disciplines ont fait la manip mais chaque discipline s'est forcée de faire en sorte que si jamais il n'y avait pas de résultats dans la manip au niveau de l'interdisciplinarité, elle trouve quelque chose à publier au niveau de sa partie à elle. Typiquement, c'est ce qu'on va faire nous. J'ai un autre projet interdisciplinaire avec une équipe de psychologie qui étudie le contrôle moteur sur la sécurité routière, on va essayer de voir s'il y a vraiment des liens entre nos deux disciplines sur un même projet. Si jamais l'hypothèse est nulle, on a fait en sorte que ma partie psycho-sociale soit publiée quand même. Je crois que pour l'instant les publications du labo, sont plutôt portées sur de l'unidisciplinarité. Il me semble »(Entretien 1).

Notons de plus que l'espace de publication dans des revues indexées par l'AERES en STAPS (domaine pourtant pluridisciplinaire) reste assez disciplinaire et même une revue clairement pluridisciplinaire comme la revue STAPS ne propose guère de recherche interdisciplinaire comme nous avons pu le montrer (Collinet, et al., 2012).
Finalement dans cet espace tensionnel, on a pu observer des figures diverses de l'interdisciplinarité. Celle-ci peut renvoyer une juxtaposition de disciplines ou à des formes de collaboration voire d'intégrations plus avancées entre les disciplines. On retrouve ici la distinction établie dans les années 1970 par Piaget entre pluri et interdisciplinarité notamment. D'autres enfin, viseraient la création d' « interdisciplines » (Frodeman, 2010) ou de transdisciplines.

Face à cette double injonction, les chercheurs doivent trouver des façons de tenir des systèmes de contraintes variées. Nos investigations ont permis de repérer quelques chemins assez frayés même s'il existe à la marge des solutions plus marginales. Cette idée de « chemins frayés » ne se superpose pas à la notion de stratégie qui suppose un espace de calcul assez stabilisé ni un ethos ou un habitus qui serait durablement structurant. Elle suggère plutôt une forme de régularité ou de construction partagées. On peut aussi la rapprocher de la notion de «stock de savoirs » chez Garfinkel (1967) ou de « stock de connaissances socialement partagé » chez Schütz (1943) dans la mesure où nous pensons que ce sont des expériences individuelles et collectives qui ont pu résister de façon durable à des contre-argumentations et à des évaluations. Mais c'est plus la notion de prise collective développée par Bessy \& Chateauraynaud (1995) pour décrire les façons dont les acteurs parviennent à échanger leurs façons de faire dans les processus d'authentification qui a inspiré cette expression qui veut désigner des chemins plusieurs fois empruntés qui s'offrent du coup comme des registres d'action identifiés. À ce titre, suivre un de ces «chemins frayés » participe à la légitimité de cette façon de faire et assure en même temps d'une certaine visibilité.

On a identifié six chemins qui permettent de répondre partiellement aux différentes injonctions, bien que nous les considérions comme un continuum, nous les avons regroupés pour l'exposé en deux groupes selon la manière dont ils se positionnement par rapport à la disciplinarité et l'interdisciplinarité.

\section{Proposer un travail interdisciplinaire en gardant une forte disciplinartié (Tab. 1)}

Chaque chemin frayé est décliné en fonction de sa position par rapport aux injonctions disciplinaire et interdisciplinaire, et des registres d'action qu'il produit. Après avoir synthétisé les chemins frayés dans un tableau nous les détaillerons et illustrerons un à un.

\subsection{Cohabiter dans un même espace de travail scientifique}

L'injonction interdisciplinaire est particulièrement forte lors des évaluations des collectifs de recherche. À défaut de pouvoir recruter des collègues ayant tous des valences disciplinaires multiples, il reste à montrer que les spécialités disciplinaires s'harmonisent parfaitement dans un 
Tableau 1. Chemin frayé : proposer un travail interdisciplinaire en gardant une forte disciplinarité.

\begin{tabular}{|c|c|c|}
\hline & $\begin{array}{l}\text { Vis-à-vis de l'injonction } \\
\text { disciplinaire }\end{array}$ & $\begin{array}{l}\text { Vis-à-vis de l'injonction } \\
\text { interdisciplinaire }\end{array}$ \\
\hline \multirow[t]{5}{*}{ Cohabiter } & Piste envisagée : & Piste envisagée : \\
\hline & $\begin{array}{l}\text { Se garder la possibilité } \\
\text { individuellement d'une } \\
\text { production disciplinaire }\end{array}$ & $\begin{array}{l}\text { Mettre en avant un collectif } \\
\text { pluridisciplinaire } \\
\text { Donner à voir une } \\
\text { harmonisation dans un travail } \\
\text { collectif ayant vocation à être } \\
\text { interdisciplinaire }\end{array}$ \\
\hline & Risques : & Risques : \\
\hline & $\begin{array}{l}\text { Détourner les objets pour } \\
\text { les partager }\end{array}$ & $\begin{array}{l}\text { Harmonisation de façade sans } \\
\text { travail interdisciplinaire }\end{array}$ \\
\hline & $\begin{array}{l}\text { Solutions : } \\
\text { Choisir des objets fourre-tout } \\
\text { ou imposer ses objets }\end{array}$ & $\begin{array}{l}\text { Solutions : } \\
\text { Trouver des liens externes } \\
\text { (thématiques, objets...) } \\
\text { suffisamment larges } \\
\text { pour être partagés } \\
\text { Inventer un lexique pour désigner } \\
\text { des « thématiques communes », } \\
\text { «des objets transversaux », } \\
\text { des « lignes de recherche »... }\end{array}$ \\
\hline \multirow[t]{5}{*}{ Se contenter d'un affichage } & Piste envisagée : & Piste envisagée : \\
\hline & Produire des articles disciplinaires & $\begin{array}{l}\text { Faire de la rhétorique } \\
\text { Afficher une posture générale } \\
\text { qui engage peu pour } \\
\text { son propre travail }\end{array}$ \\
\hline & Risques : & Risques : \\
\hline & $\begin{array}{l}\text { Inquiéter les tenants de la } \\
\text { discipline par une menace de } \\
\text { dispersion. }\end{array}$ & $\begin{array}{l}\text { Être repéré comme } \\
\text { un rhétoricien } \\
\text { Apparaître réductionniste }\end{array}$ \\
\hline & $\begin{array}{l}\text { Solutions } \\
\text { Défendre la discipline } \\
\text { en reconnaissant ses limites }\end{array}$ & $\begin{array}{l}\text { Solutions : } \\
\text { S'engager dans des promesses } \\
\text { en renvoyant à plus tard } \\
\text { les véritables contraintes }\end{array}$ \\
\hline \multirow[t]{4}{*}{ Ouvrir a minima } & Piste envisagée : & Piste envisagée : \\
\hline & $\begin{array}{l}\text { Montrer l'intérêt } \\
\text { de sa discipline }\end{array}$ & $\begin{array}{l}\text { Entreprendre une lecture un peu } \\
\text { fine et/ou discuter de façon } \\
\text { précise avec des collègues } \\
\text { de disciplines plus éloignées }\end{array}$ \\
\hline & $\begin{array}{l}\text { Risques : } \\
\text { Se disperser encore plus } \\
\text { en perdant du temps avec } \\
\text { des collègues éloignés des } \\
\text { « véritables débats» } \\
\text { de la discipline. }\end{array}$ & $\begin{array}{l}\text { Risques : } \\
\text { S'exposer à la critique sur } \\
\text { un champ dans lequel on n'est pas } \\
\text { forcément très compétent }\end{array}$ \\
\hline & $\begin{array}{l}\text { Solutions } \\
\text { Discussion critique de quelques } \\
\text { disciplines qui doit déboucher } \\
\text { sur la valorisation de sa discipline }\end{array}$ & $\begin{array}{l}\text { Solutions : } \\
\text { Multiplier les marques de prudence } \\
\text { académique ou assumer } \\
\text { une critique virulente } \\
\text { Activer le travail interdisciplinaire } \\
\text { dans des arènes où il est valorisé }\end{array}$ \\
\hline
\end{tabular}


projet/un programme/une unité interdisciplinaire. Une première façon de gérer la tension consiste à défendre une excellence disciplinaire valorisable dans des revues reconnues par les différentes disciplines et de faire état d'un assemblage pertinent pour satisfaire la demande d'interdisciplinarité. Dès lors, l'enjeu est de produire une forme d'harmonisation et suscite un travail de mise en relation.

La valorisation du travail collectif peut, ainsi, passer par l'interdisciplinarité, soit comme principe constitutif du collectif, soit comme construction active dans un but d'affichage.

« C'est un réseau pluridisciplinaire. En fait, l'idée c'est de regrouper tous les chercheurs et enseignants chercheurs et doctorants qui travaillent sur un objet en lien avec le sport, enfin qui travaillent sur un objet de recherche en lien avec le sport, ou alors sur un objet de recherche qui peut potentiellement intéressé le sport, ses pratiquants, ses intervenants, etc. C'est ça un peu le fil conducteur, enfin le, comment dire, le fond, l'objectif du dispositif et ensuite le dispositif a comme interlocuteur particulier la région et, comment dire, le réseau justement traite avec la région du soutien de celleci et de son aide face à des projets à des allocations, à des choses comme ça quoi [...]. C'est un réseau de recherche. .. c'est un réseau pluridisciplinaire régional qui s'appelle "RESPDL" : recherche et sport en pays de la Loire » (Entretien 8).

Dans l'exemple ci-dessus, le regroupement des chercheurs et le rassemblement de leurs travaux autour d'un objet commun (ici le sport) même s'ils impliquent un partage réel minimal au niveau du travail scientifique se structurent autour d'une forme (un réseau) qui permet de répondre aux appels d'offre et de gagner en visibilité pour décrocher des contrats.

L'interdisciplinarité peut aussi être construite a posteriori impliquant un véritable travail politique.

«Actuellement on a deux grands axes. [.. .] Le laboratoire s'appelle le centre d'étude des transformations des activités physiques et sportives. Il nous fallait un concept qui puisse éventuellement être commun à tout le monde, donc le concept de transformation nous semblait intéressant. Donc on est dans l'axe 1 autour des orientations de type plutôt méthodes sociologiques - management. Et donc ça c'est un axe avec deux thématiques assez distinctes, malheureusement il faut le dire, une autour des études de type sociologique et une autour du management pur et je détaillerai éventuellement si vous le souhaitez. Et puis de l'autre côté donc une thématique autour de notion d'optimisation de la performance, orientée on va dire un peu psycho, psychophysiologie et une partie biomécanique - physiologie, et un autre... un autre axe, une autre thématique à l'intérieur du même axe autour de la perception, mais aussi de la biomécanique, donc il s'agit de croiser des grandes thématiques. Bon, il faut faire du lien, on essaie mais rassembler des entités éparses c'est difficile. Trouver des thèmes rassembleur dans lesquels chacun puisse s'intégrer » (Entretien 9).

Dans ce cas précis, la reconnaissance d'un laboratoire et son évaluation poussent les chercheurs à établir du lien entre leurs travaux personnels. L'enjeu étant de trouver des thématiques dans lesquelles chacun puisse «se caser » et définir ainsi des axes au sein du laboratoire et du même coup une éventuelle identité. Ce cas de figure est assez fréquent dans le domaine des sciences du sport en STAPS du fait des caractéristiques pluridisciplinaires de cette section. Les besoins d'enseignement peuvent conduire à recruter des titulaires spécialistes de toutes les disciplines. Mais du point de vue de la recherche, ces chercheurs issus de disciplines différentes n'ont pas toujours l'habitude de travailler ensemble (dans l'exemple précédent un sociologue se trouve dans le même axe que les physiologistes). On perçoit cependant dans l'extrait précédent combien l'exercice est délicat ( «malheureusement », «il faut faire du lien »), la difficulté réside dans le constitution de liens entre des recherches qui se sont constituées séparément les unes des autres. Les solutions sont alors souvent de trouver des thématiques très générales (comme le risque, le bien-être ...) et le risque est toujours de juxtaposer les travaux sans partage ni discussion.

\subsection{Viser un affichage interdisciplinaire}

Dans les productions scientifiques étudiées, de nombreux textes ne font qu'une allusion superficielle à l'interdisciplinarité, essentiellement au moment du cadrage théorique et souvent pour insister sur la pluralité des travaux portant sur leur objet de recherche comme, par exemple, l'article de Chantelat faisant un bilan de la littérature socio-économique sur le sport et évoquant des travaux interdisciplinaires :

«Dans un second temps, le regroupement des textes croise deux critères permettant de rendre compte des grandes orientations développées au sein des textes : [...] l'orientation disciplinaire de l'explication en dernière instance du phénomène sportif : économie (politique, normative, ...), sociologie (économique, critique ...), sociologie et économie, voire interdisciplinarité plus large » (Chantelat, 1996).

D'autres articles se positionnent de manière plus prononcée sur l'interdisciplinarité. Mais il s'agit alors davantage de défendre un projet que de la mettre en pratique. Elle peut ainsi être présentée comme nécessaire pour mieux éclairer certains objets :

«Les progrès viendront d'échanges interdisciplinaires qui seuls permettront d'échapper aux illusions liées aux centrations uniques, de relativiser 
certaines positions, d'unifier les savoirs "régionaux" pour aboutir à une vision globale, sinon totale, nécessairement transdisciplinaire, des phénomènes agressifs » (Pfister, 1980).

Cette opération de communication peu coûteuse comporte assurément beaucoup d'avantages et présente parallèlement peu de risque. On a identifié plusieurs textes dans lesquels l'interdisciplinarité s'exprime de façon minimaliste. Les auteurs réussissent ainsi à défendre leur propre discipline puisque le propos est principalement inscrit dans une seule discipline. Seule une introduction ou une conclusion fait état de l'interdisciplinarité qui est souvent célébrée mais non déployée. Ainsi, évite-ton de s'engager dans un travail lourd sur des concepts, des méthodes ou des analyses de spécialités éloignées. Un échange lors d'un colloque ou un parcours rapide des résumés de communication suffit pour évoquer l'intérêt d'autres disciplines sur son objet qui va être redéfini dans une approche dont l'auteur est spécialiste. En conclusion, il s'agit souvent d'une promesse. Les limites de l'étude permettent d'envisager d'autres investigations dans lesquelles les autres disciplines ont potentiellement toutes leur place. Cette ouverture, qui peut se décliner comme une forme d'œcuménisme, permet également d'anticiper les accusations de réductionnisme; en assumant le côté partiel de la contribution, on fait accepte la complexité tout en défendant la discipline.

Le caractère velléitaire de cet appel à l'interdisciplinarité peut toutefois être l'objet de critique. On peut reprocher aux chercheurs s'engageant dans cette voie une célébration naïve de l'interdisciplinarité mais aussi de ne proposer que de la rhétorique. Le risque pour une recherche d'être qualifiée de «fausse interdisciplinarité » n'est pas nul et les chercheurs peuvent éprouver la nécessité de donner quelques gages de leur plus grand engagement dans l'interdisciplinarité. Les rapports étudiés portent la marque de ce doute :

\begin{abstract}
«On note une volonté d'articuler l'approche sociologique et celle des sciences de gestion. Si la cohérence globale des thématiques est à souligner, $i l$ est toutefois dommage que le descriptif des travaux soit un peu bref sans mettre en lumière les fondements théoriques des approches » (Rapport AERES, Novembre 2010).
\end{abstract}

\subsection{S'engager dans une ouverture interdisciplinaire}

L'opération est assurément plus coûteuse que la précédente. Une voie possible consiste à réaliser une revue de questions interdisciplinaire. Qu'il s'agisse de dossiers ouverts ou non, l'objet est décrit dans ses différentes approches. On mesure le coût d'un tel travail qui suppose de lire et de discuter des disciplines différentes que celles pratiquées par le chercheur. Celui-ci peut être distribué entre plusieurs collègues mais à terme, la recherche n'engage ici qu'une seule discipline. On peut également réduire l'ambition d'une revue de questions généralisée en engageant une discussion entre deux disciplines. Mais quoiqu'il en soit, il faut donc pouvoir assumer l'intérêt de l'approche privilégiée et donc disqualifier les autres en pointant des angles morts.

Cette opération conduit à prendre des risques. En s'aventurant sur des territoires moins connus, on s'ouvre à une critique violente d'une incompétence. L'anticipation de ces reproches peut conduire soit à multiplier les marques de prudence académique - que l'on retrouve pour modaliser les énoncés indexés à des hypothèses fortes soit à assumer la posture provocatrice qui consiste à s'attaquer frontalement à des approches pour poser son propos.

Une manière de réduire les risques consiste à activer l'interdisciplinarité dans des arènes qui la valorise d'une part ou qui permette de l'éprouver sans passer par les fourches caudines d'une évaluation :

«Mensuellement, on a des séminaires pluridisciplinaires sur des thématiques épistémologiques ou méthodologiques.

Et, d'autre part, on essaie de développer des travaux à travers des grandes thématiques qui permettent à différents membres et à différentes disciplines d'y contribuer sur les différentes $A N R \gg$ (Entretien 3).

Ainsi les séminaires comme les réponses à des appels d'offre permettent l'activation d'une ouverture entre disciplines sans que celle-ci puisse être soumise à des critiques trop violentes et à un manque d'effectivité dans la production de résultats.

\section{S'engager davantage dans le travail interdisciplinaire en s'éloignant des exigences disciplinaires (Tab. 2)}

Dans les chemins frayés qui suivent, l'engagement dans l'interdisciplinarité est plus forte. Les exigences disciplinaires se transforment alors, il s'agit de composer entre plusieurs disciplines pour produire des connaissances nouvelles ou plus radicalement de rompre avec une discipline de référence pour proposer la création d'une nouvelle discipline.

\subsection{Travailler sur un objet-frontière}

Nous avons pu observer que certains objets se prêtaient particulièrement à l'analyse interdisciplinaire. C'est le cas notamment de la performance ou l'entraînement :

«Le contrôle de l'entraînement est souvent réalisé à l'aide de différents indices tels que les variables physiologiques les critères de l'intensité d'exercice (Hawley, et al. 1997; Smith, et al. 
Tableau 2. Chemin frayé : s'engager d'avantage dans le travail interdisciplinaire en s'éloignant des exigences disciplinaires.

\begin{tabular}{|c|c|c|}
\hline & $\begin{array}{l}\text { Vis-à-vis de l'injonction } \\
\text { disciplinaire }\end{array}$ & $\begin{array}{l}\text { Vis-à-vis de l'injonction } \\
\text { interdisciplinaire }\end{array}$ \\
\hline \multirow[t]{3}{*}{$\begin{array}{l}\text { Travailler sur des objets } \\
\text { frontières }\end{array}$} & $\begin{array}{l}\text { Piste envisagée : } \\
\text { Étendre sa disciplin }\end{array}$ & $\begin{array}{l}\text { Piste envisagée : } \\
\text { Un travail collectif rigoureux } \\
\text { sur } 2 \text { ou } 3 \text { discipline } \\
\text { Produire des connaissances nouvelles, } \\
\text { mieux rendre compte 'un objet d'étud }\end{array}$ \\
\hline & $\begin{array}{l}\text { Risque : } \\
\text { Ne pas être compris dans sa discipline } \\
\text { et se faire marginalise }\end{array}$ & $\begin{array}{l}\text { Risque : } \\
\text { Se faire critiquer des deux côté } \\
\text { Ne pas se faire publie }\end{array}$ \\
\hline & $\begin{array}{l}\text { Solution : } \\
\text { Multiplier des marqueurs } \\
\text { d'appartenance disciplinaire } \\
\text { (en tout cas dans la discipline de réfé- } \\
\text { rence) }\end{array}$ & $\begin{array}{l}\text { Solution : } \\
\text { Défendre l'interdisciplinarit }\end{array}$ \\
\hline \multirow[t]{3}{*}{$\begin{array}{l}\text { Défendre une discipline } \\
\text { interdisciplinaire }\end{array}$} & $\begin{array}{l}\text { Piste envisagée : } \\
\text { Défendre l'existence d'une } \\
\text { discipline peu légitime } \\
\text { Façonner un nouveau } \\
\text { champ scientifique }\end{array}$ & $\begin{array}{l}\text { Piste envisagée: } \\
\text { Attaquer le réductionnisme discipli- } \\
\text { naire } \\
\text { Insister sur l'adaptation de la disci- } \\
\text { pline } \\
\text { aux objets complexes et/ou pratiques }\end{array}$ \\
\hline & $\begin{array}{l}\text { Risques : } \\
\text { Ne pas exister } \\
\text { Ne pas se faire reconnaître } \\
\text { en tant que discipline scientifique }\end{array}$ & $\begin{array}{l}\text { Risques: } \\
\text { Se faire disqualifier par les } \\
\text { disciplines canoniques } \\
\text { Être marginalisé }\end{array}$ \\
\hline & $\begin{array}{l}\text { Solutions : } \\
\text { Faire exister une communauté } \\
\text { épistémique } \\
\text { S'ériger en spécialiste } \\
\text { Donner des garanties de son } \\
\text { approche scientifique }\end{array}$ & $\begin{array}{l}\text { Solutions : } \\
\text { Louvoyer entre une critique de } \\
\text { l'académisme et une volonté de légiti- } \\
\text { mer } \\
\text { une discipline } \\
\text { En appeler à la demande sociale }\end{array}$ \\
\hline \multirow[t]{3}{*}{$\begin{array}{l}\text { Revendiquer une } \\
\text { transdisciplinarité }\end{array}$} & $\begin{array}{l}\text { Piste envisagée : } \\
\text { Dépasser les frontières } \\
\text { disciplinaires }\end{array}$ & $\begin{array}{l}\text { Piste envisagée : } \\
\text { Valoriser la transdisciplinarité }\end{array}$ \\
\hline & $\begin{array}{l}\text { Risques : } \\
\text { Ne pas être compris }\end{array}$ & $\begin{array}{l}\text { Risques: } \\
\text { Ne pas être discuté } \\
\text { (marginalisation) }\end{array}$ \\
\hline & $\begin{array}{l}\text { Solutions: } \\
\text { Disqualifier le réductionnisme } \\
\text { disciplinaire }\end{array}$ & $\begin{array}{l}\text { Solutions : } \\
\text { Défendre une position } \\
\text { épistémologique forte } \\
\text { Convocation de la demande } \\
\text { sociale (enseignement de l'EPS) }\end{array}$ \\
\hline
\end{tabular}

1999 et Hoogeveen, 2000) et/ou les variables psychologiques (Borg, 1970). Mais peu d'auteurs se sont intéressés à une approche pluridisciplinaire des effets de l'entrâ̂nement » (Delattre, Garcin, Mille-Hamard, \& Billat, 2003).

Ou d'objets précis comme la mesure du « coût énergétique $\gg^{12}$ :

«Dans cette optique, nous partagerons a priori le point de vue de Mc Mahon (1984) selon lequel, pour comprendre le fonctionnement des muscles,

\footnotetext{
12 Le coût énergétique représente la quantité d'énergie dépensée par unité de temps pour exécuter une action.
}

$i l$ est tout aussi utile, grâce aux regards croisés que permettent la physique et la biologie, d'accorder une place au point de vue mécanique autant qu'aux points de vue biochimique et histologique » (Thys, 2001).

L'article de Thys, titré «Place de l'énergie mécanique dans le déterminisme du coût énergétique de la locomotion », propose une approche dans laquelle sont analysées les contributions de paramètres mécaniques du mouvement sur une variable biologique, le coût énergétique, lors de la marche et de la course. Une analyse similaire opérée sur les différentes combinaisons de disciplines laisse apparaître un certain nombre de ces « objets-frontières » 
interdisciplinaires : le 《 coût énergétique », mais aussi les notions connexes de « rendement » ou de «fatigue 》 (croisant biomécanique et physiologie), le «stress » (psychologie et physiologie), les « coordinations motrices » (psychologie et biomécanique) ou encore l' « imagerie mentale » (psychologie et neurosciences). Parfois, il s'agit d'analyser un phénomène à travers différents niveaux de description. Dans d'autres articles, il s'agit d'étudier l'influence de facteurs liés à un domaine disciplinaire sur d'autres facteurs liés à un autre domaine. Dans d'autres cas encore, il s'agit d'objectiver un niveau de description difficilement observable à partir de variables et de facteurs mobilisés dans une autre discipline. C'est par exemple le cas des études sur le stress dans lesquelles des indicateurs physiologiques sont les témoins de processus psychologiques inobservables.

Ce chemin est particulièrement coûteux. Du point de vue des contraintes interdisciplinaires, il suppose un travail rigoureux engageant deux ou trois disciplines et comporte plusieurs risques. L'un consiste à s'ouvrir à la critique émanant des gardiens des différentes disciplines. La sanction peut être particulièrement lourde car de telles recherches peuvent avoir de réelles difficultés à se faire publier dans des revues disciplinaires en raison d'un défaut d'inscription claire.

Ce type de recherche positionne le chercheur dans un espace de travail interdisciplinaire qui n'est pas toujours facile à valoriser d'un point de vue scientifique. Ainsi un chercheur interrogé qui se livre à ce type de travaux pouvait signaler :

\section{«Mes thématiques de recherche ce sont les mar- queurs de fatigue, enfin au départ c'était les mar- queurs de fatigue du surentraînement, en couplant des marqueurs physiologiques et de marqueurs psy- chologiques [...]. On se fait critiquer des deux cô- tés. Quand je vais dans des congrès physiologiques, je me fais un peu critiquer parce que j'amène de la psycho, et pareil quand je vais dans des congrès de psycho je me fais critiquer parce que je ne suis pas estampillée psycho »(Entretien 12).}

Cet entretien illustre bien le problème de la tension entre l'interdisciplinarité comme mot d'ordre et l'interdisciplinarité en acte, celle-ci restant difficile à valoriser sur la scène académique et notamment sur celle de l'évaluation du travail scientifique.

Pour surmonter ces risques, les chercheurs peuvent mobiliser plusieurs leviers. D'une part, ils peuvent s'efforcer de faire la preuve de leur « légitimité» dans leur propre discipline. Cela peut passer par la multiplication de marqueurs d'appartenance disciplinaire (en tout cas dans la discipline de référence) : dans nos entretiens, des chercheurs précisent combien la citation d'un auteur ou d'une publication dans une revue donnée participent d'une volonté de donner des gages disciplinaires. Une solution consiste à produire, à la marge de la recherche considérée, d'autres études s'inscrivant pleinement dans le champ de référence mais le temps nécessaire pour travailler sur des objets-frontières interdit très souvent aux chercheurs de mener d'autres projets. D'autre part, ils peuvent s'engager vivement dans une défense de l'interdisciplinarité. Promouvant la cause, dénonçant les contraintes qui se dressent à ceux qui font « réellement» de l'interdisciplinarité, ils s'efforcent de faire montre de l'étendue de leur érudition dans plusieurs disciplines. Une étape supplémentaire consiste à participer à l'institutionnalisation de cette posture.

\subsection{Défendre une « discipline interdisciplinaire »}

Plusieurs disciplines se situent à un carrefour entre plusieurs autres. C'est le cas notamment de la didactique (Collinet, et al., 2013). Elle partage de nombreux objets avec les sciences sociales (représentation, normes, identité professionnelle...), mais surtout avec la psychologie (perception, motivation, estime de soi, leadership et apprentissage...). Un enseignant chercheur en didactique précise ses tentatives de «travailler en psycho-sociologie et parfois, je côtoie un peu de sociologie qualitative » (Entretien 19), témoignant ainsi d'une position intermédiaire. Les chercheurs revendiquant une inscription dans le domaine de la didactique se définissent surtout à partir des finalités de la recherche didactique qui consisteraient prioritairement à formuler des prescriptions explicites pour l'action ainsi qu'au niveau des protocoles de recherche en restant attachés à une inscription dans un espace écologique.

Qu'il s'agisse de «management», de « didactique » ou de « préventologie », il faut, dans cette perspective, participer à la création ou à la défense d'une discipline peu légitime. Pour cela, il convient de produire des efforts qui visent à faire exister une communauté épistémique instituée; cela suppose par exemple la création d'une ou des revues, que l'on peut identifier par un lexique disciplinaire (dénoncé comme étant un jargon), l'organisation de colloques, la création d'une société savante ${ }^{13}$ etc.; dans un second temps, on pourra se référer à des débats canoniques instituant cette discipline, raconter son histoire...

Du point de vue de l'interdisciplinarité, on cherchera ici à attaquer le réductionnisme disciplinaire. Ce sont les disciplines académiques qui sont visées notamment lorsqu'elles prennent pour cible ces velléités disciplinaires. La tâche n'est pas aisée car elle consiste à louvoyer entre une critique de l'académisme et une volonté de légitimer une discipline. Une des voies possibles tient dans la convocation de l'histoire des sciences ou de l'épistémologie. Il s'agit alors de produire une sorte de méta connaissance sur les savoirs scientifiques eux-mêmes qui se questionne sur les STAPS en tant que discipline universitaire, leur organisation, leur fonctionnement et les modes de travail scientifique à l'œuvre dans un domaine par nature pluridisciplinaire. On peut repérer des analyses épistémologiques qui discutent les formes d'interdisciplinarité à

\footnotetext{
13 Comme l'ARIS : Association pour la Recherche pour l'Intervention en Sport.
} 
l'œuvre dans les sciences du sport et leurs enjeux. «Qu'en est-il ? Y a-t-il ou non un objet spécifique, des objets spécifiques ou bien des traditions de recherches et des paradigmes particuliers aux STAPS? Les STAPS sont-elles une science, un savoir pluridisciplinaire, des rationalités hétérogènes ou bien une science plurielle? 》, se demande Jarnet (2004).

Mais c'est en convoquant la demande sociale et/ou la complexité pratiques des phénomènes que l'on peut le plus facilement légitimer ces approches. Les enjeux de l'obésité ou de la lutte contre le dopage, par exemple, supposent de mobiliser des acteurs qui connaissent toutes les dimensions du problème sans le réduire.

\subsection{Défendre la transdisciplinarité}

Figure emblématique des STAPS, la volonté de créer une science de la motricité figure parmi les positions avancées par certains chercheurs regroupés sous la bannière de la praxéologie motrice.

\begin{abstract}
«Définir un objet propre, caractérisé par une pertinence originale, est une nécessité pour accéder au statut de discipline scientifique. Aucune science n'a pris l'action motrice pour objet propre. Le champ est donc libre.

Il s'agit de chercher à comprendre comment se prennent les décisions dans l'action, en fonction des caractéristiques des jeux, des sports, souvent plus complexes que celles du travail, en raison de la présence directe d'adversaires, par exemple. La réussite passe par l'intégration de données concernant aussi bien, le rapport à l'espace et aux objets, le rapport au corps propre, que les diverses formes du rapport à autrui : on est bien dans une perspective transdisciplinaire »(Parlebas, 2005).
\end{abstract}

En prétendant unifier toutes les disciplines des sciences du sport sous une seule science, elle semble la seule à tenir une version transdisciplinaire qui fonctionne par une disqualification de disciplines habituelles.

\footnotetext{
«Les UFR - STAPS, autre fois Instituts Régionaux d'Education Physique, ont une histoire marquée par de graves problèmes d'identité. Jusqu'à il y a une trentaine d'années s'y développait une multidisciplinarité fondée sur les sciences biologiques. Depuis, sont venues s'ajouter les sciences humaines et sociales. De fait, les cursus proposés s'inscrivent aujourd'hui dans une pluridisciplinarité largement ouverte, qui aboutit, compte tenu de l'originalité des situations prises en compte par les chercheurs à des apports souvent intéressants. Notons que cette pluridisciplinarité demande aux étudiants de solides facultés d'adaptation. Quelques équipes, rares, travaillent dans une perspective interdisciplinaire : les résultats sont ici peu nombreux et souvent décevants. Il arrive en effet que
}

certains chercheurs masquent sous une interdisciplinarité affichée confusions et faiblesses : l'addition de deux demi-compétences ne donne pas une compétence entière!

De toutes manières, ni la pluridisciplinarité ni l'interdisciplinarité n'ont la capacité de fournir un fil directeur, une identité originale aux UFR $S T A P S »$ (id. p. 34).

Le coût de cette posture est très lourd car elle suppose à la fois de valoriser politiquement cette transdisciplinarité en dévalorisant le réductionnisme des autres et en défendant une position épistémologique forte. Cela suppose d'affronter beaucoup de protagonistes sur leur propre terrain.

Le principal risque n'est pas tant d'être considéré comme incompétent car il est toujours possible de déplacer l'épreuve au nom du réductionnisme de celui qui porte la critique en mobilisant des ressources dont il ne dispose pas, mais d'être marginalisé. Le conflit entre Parlebas et Vigarello étendu à d'autres acteurs (Gonzales, 2013) en témoigne. Dès lors, c'est une quête d'existence qui structure ce chemin, qui passe par un repli identitaire (sauver les STAPS) et une mobilisation de l'histoire des sciences.

Cette proposition de qualification en termes de « chemins frayés» ne signifie pas que tous les acteurs les empruntent et les reconnaissent. D'une part, parce qu'il est toujours possible de tenter des combinaisons plus ou moins sophistiquées, défendant par exemple sa discipline et en s'impliquant partiellement dans un projet interdisciplinaire assez lourd. Les combinaisons les plus audacieuses présentent un autre risque : on peut se voir reprocher de se contredire. Une carrière de chercheur fonctionne sur la réputation qui porte sur des indicateurs mais aussi et surtout sur des qualifications (X travaille sur ceci, il est spécialiste de ...). À partir d'un certain seuil, l'engagement dans l'interdisciplinarité peut participer à une réputation qui rend difficiles les autres expérimentations qui pourraient conduire à se voir reprocher de se contredire.

Cette tension repose essentiellement sur la question de l'évaluation et les débats qu'elle suscite. En effet, une contrainte de cohérence invite à ce que la défense d'une position s'accompagne de gages. Il est par exemple difficile d'inviter à la bienveillance lors d'une expertise d'un dossier se voulant interdisciplinaire, si l'évaluateur ne vise que l'excellence disciplinaire. Cette exigence conduit à lier des pratiques scientifiques à la défense de politiques scientifiques.

\section{Conclusion}

L'interdisciplinarité apparaît comme une figure complexe dans le domaine qui nous intéresse. C'est à la fois une sorte de matrice originelle au fondement de la discipline, un mode de fonctionnement à privilégier, gage de la spécificité des STAPS, mais en même temps elle reste difficile 
à tenir et difficilement porteuse d'excellence académique reconnue.

Cette tension pousse les chercheurs à différents modes d'investigations du plus léger au plus contraint comme le montrent les chemins frayés mis au jour. Sachant que les formes les plus « dures» à visée transdisciplinaire sont l'objet de vives critiques au sein de la communauté et souffrent parfois d'une forme d'ostracisme (la praxéologie parlebasienne par exemple) et d'une remise en question de leur scientificité ou de leur intérêt scientifique.

Les tensions soulevées poussent aussi les enseignantschercheurs à investiguer plusieurs registres de travaux navigant entre des recherches et des actions interdisciplinaires et d'autres plus typiquement disciplinaires. Une analyse plus précise des différents espaces de recherche devraient permettre de mieux circonscrire les arènes scientifiques les plus propices à l'interdisciplinarité (certains appels à projet par exemple) alors que d'autres restent plus exclusivement disciplinaires (les évaluations dans les comités de sélection par exemple).

De la même manière, les évaluations oscillent entre plusieurs postures en soulignent l'intérêt en même temps qu'elles en pointent les limites ou les insuffisances du travail interdisciplinaire et soulignant tantôt l'absence de fondements théoriques suffisamment solides, tantôt la difficulté de partage des concepts ou des méthodes.

Finalement, l'interdisciplinarité est souvent associée à une promesse et l'existence de dialogues pluridisciplinaires, de compétences multidisciplinaires voire de projets ou de démarches interdisciplinaires renvoient alors à un futur (Trabal, et al., 2014). La projection dans le futur d'une interdisciplinarité à venir et à penser permet de réduire les tensions repoussant à plus tard la mise en chantier de son opérationnalité.

Nous pouvons noter en outre, que ces tensions ont à voir avec des débats politiques qui ont toujours traversé la science. Les enjeux sont pluriels, épistémologiques pour certains (il s'agit de mieux lire le réel et de mieux éclairer certains objets complexes), politiques pour d'autres (en proposant une recherche plus directement socialement utile). La politisation de la science d'une part et sa managérialisation d'autre part portent le travail scientifique vers la réponse à de grands enjeux sociétaux dont chaque discipline prise isolément ne peut s'emparer et donner le gage de son efficacité sociale. Néanmoins les tensions sont vives comme nous l'avons vu et les injonctions paradoxales; les chemins frayés, en tant que régimes d'action stabilisés, permettent d'y répondre en offrant des voies de travail partagées et modulables quant à l'engagement dans l'interdisciplinarité.

\section{Bibliographie}

Bessy, C., \& Chateauraynaud, F. (1995) [2014]. Experts et faussaires - pour une sociologie de la perception. Paris : Métailié.
Boisot, M. (1972). Discipline et interdisciplinarité. In $O C D E$, L'interdisciplinarité, problème d'enseignement et de recherche dans les universités (pp. 90-97). Paris : Rapport pour l'Organisation de Coopération et de développement économiques.

Chantelat, P. (1996). La littérature socio-économique du sport en France : essai de classification et questionnement épistémologique. STAPS, 41, 55-75.

Chateauraynaud, F. (2003). Prospéro. Une technologie littéraire pour les sciences humaines. Paris : Éditions du CNRS.

Collinet, C. (2000). La recherche en STAPS. Paris : PUF.

Collinet, C. (2001). EPS et sciences. Paris : PUF.

Collinet, C., Trabal, P., \& Delalandre, M. (2013). Forms and modes of apprehending interdisciplinarity, A socio-computer analysis of sports sciences. Bulletin of Sociological Methodology/Bulletin de Méthodologie Sociologique 119, 1, 61-78.

Collinet, C., Terral, P., \& Trabal, P. (2012). La Revue STAPS et l'interdisciplinarité : une étude socio-informatique ». STAPS, 96-97, 29-47.

Delattre, E., Garcin, M., Mille-Hamard, L., \& Billat, V. (2003). Analyse objective et subjective des effets de l'entraînement chez des demi-fondeurs et des cyclistes. in $10^{\mathrm{e}}$ congrès de l'ACAPS, Toulouse : 30, 31 octobre et 1 novembre, $238-239$.

Escriva, J.-P. (2008). L'improbable interdisciplinarité des sciences et techniques des activités physiques et sportives. STAPS, 93, 114-118.

Frodeman, R., Thompson Klein, J., Mitcham, C., (Eds.). (2010). The Oxford Handbook of Interdisciplinarity. Oxford: Oxford University Press.

Garfinkel, H. (1967). Studies in Ethnomethodology. PrenticeHall: Englewood.

Garnier, S., Mejean, L., \& Minotti, C. (1990). Influence des activités physiques et sportives sur l'alimentation des femmes incarcérées. STAPS, 23, 51-56.

Gonzales, O. (2013). La création d'une science des activités physiques et sportives : entre controverse épistémologique et querelle de famille. In Collinet C. \& Terral P., (Eds.), Sport et Controverses. Paris : Edition des archives contemporaines.

Heckhausen, H. (1972). Discipline et interdisciplinarité. In OCDE, L'interdisciplinarité, problème d'enseignement et de recherche dans les universités. Paris : Rapport pour l'Organisation de Coopération et de développement économiques.

Jarnet, L. (2004). Pour une épistémologie aposterioriste des STAPS. STAPS, 65, 27-41.

OCDE, 1972. L'interdisciplinarité, problème d'enseignement et de recherche dans les universités. Paris : Rapport pour l'Organisation de Coopération et de développement économiques.

Parlebas, P. (2005). Rencontre avec...». Carrefours de l'éducation, 20, 89-96. 
Pfister, R. (1980). La mesure de l'agressivité. STAPS, 2, $23-33$

Piaget, J. (1972). L'épistémologie des relations interdisciplinaires. In Apostel L. (Ed.), L'interdisciplinarité : problèmes d'enseignement et de recherche dans les universités. Paris : Rapport pour l'Organisation de Coopération et de développement économiques.

Prud'homme, T., Gingras, Y., Couillard, A., \& Terrasson, D. (2012). Les mesures de l'interdisciplinarité. Pratique et attitudes dans un centre de recherche français. l'IRSTEA, Montréal, CIRST, http://www.cirst.uqam.ca/Portals/0/ docs/note_rech/2012-01.pdf.

Schütz, A. (1943). The Problem of Rationality in the Social World. Economica New Series 10, 38, 130-149.

Sinaceur, M.A. (1992). Quelques réflexions sur l'interdisciplinarité. In Portella E., (Ed.), Entre savoirs : l'interdisciplinarité en acte. Enjeux, obstacles, résultats. Toulouse : Erès.

Sperber, D. (2010). Pourquoi repenser l'interdisciplinarité ? In Origgi, G., Darbellay, F., (Ed.), Repenser l'interdiscplinarité (pp. 19-30). Genève : Slatkine.

Stehr, N., \& Weingart, P. (2000). Practising Interdisciplinarity. Toronto: University of Toronto Press.
Terral, P. (2003). La construction sociale des savoirs du monde sportif : sociologie des conceptions. Thèse de $3^{\mathrm{e}}$ cycle en sociologie non publiée, Université Paris IV - Sorbonne.

Thibault, J. (1981). Recherche historique et activités physiques et sportives. Revue STAPS, 3, 33-38.

Thys, H. (2001). Place de l'énergie mécanique dans le déterminisme du coût énergétique de la locomotion. STAPS, 54, 131-143.

Trabal, P., Collinet, C., Terral, P. (2014). Le travail scientifique interdisciplinaire : réalités et contraintes. Rapport pour l'Agence Nationale de la Recherche, Université Paris Ouest Nanterre.

Vanpoulle, Y. (2011). Connaissance par corps et épistémologie en STAPS. STAPS, 92, 75-90.

Vinck, D. (2000). Pratiques de l'interdisciplinarité. Mutation des sciences, de l'industrie et de l'enseignement. Grenoble: Presses Universitaires de Grenoble.

Weingart, P. (2010). A Short History of Knowledge Formations. In Frodemann R., Thomson Klein J., \& Mitcham C., (Eds). The Oxford Handbook of Interdisciplinarity (pp. 3-14). Oxford: Oxford University Press. 